\title{
Incidence and risk factors associated with surgical site infection following cesarian section at Kibungo Referral Hospital, Rwanda - A prospective cohort study
}

\author{
Authors: D. Akimana ${ }^{1, *}$; C. Mpirimbanyi²; O. Ituze ${ }^{2}$; F. F. Uwera ${ }^{1}$; E. Rutayisire ${ }^{1}$ \\ Affiliations: ${ }^{1}$ Department of Public Health, School of Health Sciences, Mount Kenya University, \\ Kigali, Rwanda; ${ }^{2}$ Department of Surgery, Kibungo Referral Hospital, Eastern Province, Rwanda
}

\begin{abstract}
INTRODUCTION: Cesarian section (CS) is lifesaving both for the mother and the baby. Worldwide, there has been an increase in the incidence of CS. However, complications may arise postoperatively for both mother and newborn.

Our aim was to determine the incidence rate of post-CS surgical site infection (SSI), identify factors associated with SSI, and identify the most frequent microorganisms associated with the presence of post-CS SSI.
\end{abstract}

METHODS: This is a prospective cohort study conducted at $\mathrm{KRH}$, including all CS, performed from February to April 2020. Patient's demographics, operative management, and outcomes were analyzed.

RESULTS: A total of 201 patients aged between 15 to 47 years were operated on and $3.48 \%$ developed SSI. 90\% were from Ngoma district, 47\% had secondary education followed by $36 \%$ with primary education. The majority $(97 \%)$ had no comorbidities. Povidone and chlorhexidine combined was the most commonly used disinfectant. 53\% were emergencies and $92 \%$ of CS were performed by general practitioners. The average duration of operation was between 30 to 45 minutes. Showering prior to operation $(R R=0.39)$ at $95 \% \mathrm{Cl}[0.005-0.29]$, not shaving 30 minutes prior to incision (RR: 25.5 ) at $95 \% \mathrm{Cl}$ [3.5-18.7] and use of both povidone and chlorhexidine for skin preparation $(\mathrm{RR}=0.15)$ at $95 \% \mathrm{Cl}[0.1-1.6]$ are associated with reduced risk of developing SSI. Obstructed labor/dystocia ( $(R R=4.55)$ at 95\%, Cl [1.6-45.4]) increases the infection risk. Staphylococcus aureus was the most frequently isolated microorganism in post-CS SSI patients.

CONCLUSION: Active hospital infection services and adherence to evidence-based guidelines for SSI prevention measures would reduce the post-CS SSI incidence rate and improve patient care.

Keywords: Cesarian section, Incidence, Outcome, Microorganisms, Surgical Site Infection

\section{INTRODUCTION}

A review of the literature highlights that surgical site infections are a common major complication after caesarian sections worldwide and are mainly responsible for increased maternal morbidity and

\footnotetext{
*Corresponding author: Delphin Akimana, Department of Public Health, School of Health Sciences, Mount Kenya University Rwanda Email: delphinakimana21@gmail.com, Telephone: +250784856700; Potential Conflicts of Interest (Col): All authors: no potential conflicts of interest disclosed; Funding: All authors: All authors: no funding has been sought or gained for this project; Academic Integrity. All authors confirm that they have made substantial academic contributions to this manuscript as defined by the ICMJE; Ethics of human subject participation: The study was approved by the local Institutional Review Board. Informed consent was sought and gained where applicable; Originality: All authors: this manuscript is original has not been published elsewhere; Review: This manuscript was peer-reviewed by three reviewers in a double-blind review process; Type-editor: Gilson (USA).
}

Received: $13^{\text {th }}$ June 2021; Initial decision given: $31^{\text {st }} J$ uly 2021; Revised manuscript received: $02^{\text {nd }}$ August 2021; Accepted: $25^{\text {th }}$ November 2021 Copyright: (C) The Author(s). This is an Open Access article distributed under the terms of the Creative Commons Attribution License (CC BY-NC-ND) (click here) which permits unrestricted use, distribution, and reproduction in any medium, provided the original work is properly cited. Publisher: Rwanda Biomedical Centre (RBC)/Rwanda Health Communication Center, P. O. Box 4586, Kigali. ISSN: 2079-097X (print); 2410-8626 (online) 
mortality, dissatisfaction of the patient and family, longer hospital stay, and higher treatment cost $[1,2]$. The infection can come from the break of skin normal flora, spillage from the gastrointestinal system, or others.

The incidence of post-CSSSI varies from high income to low and middle-income settings. In developed country, SSI results in morbidity between 3\% to $16 \%$ and death $0.3 \%$ to $0.8 \%$ [3]. Compared to lowincome and Middle-income settings, incidence can go up to $25 \%[4,5,6]$. In Rwanda, it was more slightly lower at $4.9 \%$ compared to other countries [2]. This wide variation is due to advanced hospital infection control services and good implementation of evidence-based guidelines for SSI prevention measures [7].

The occurrence of SSI depends on the complex interaction of such as the pre-morbid health condition of the mother, nutritional status, age of the mother, the presence of prolonged premature rupture of the membrane, and prolonged duration of CS more than an hour or surgical technique used $[3,5,8,9]$.

Staphylococcus aureus, Escherichia coli, Klebsiella pneumonia, and pseudomonas aeruginosa are the most common isolates of post-CS SSI. However, staphylococci, being a major component of the skin microbiome, remain the most common microorganism responsible for post-CS SSI $[2,5,6,9,10]$. Moreover, the microbiology patterns in organ/space occupying infections for post-CS peritonitis are polymicrobial and are dominated by E. coli (25\%) and Klebsiella pneumonia (21\%) [11].

\section{METHODS}

Study design: A prospective cohort study. The study was conducted at Kibungo Referral Hospital, one of the new three newly upgraded hospitals to tertiary referral hospital level in Rwanda, located in the Eastern Province. It originally serves a catchment area of approximately 400,000 population and has a capacity of 312 beds. There are 3 operating suites and the gynecology and obstetrics ward have 73 beds. An estimated 2500 operations are performed annually. The most common operation is the cesarian section (72\%).

Participants: The study included all women aged 15 years and above who gave birth by caesarian section, both elective and emergency, at Kibungo Referral Hospital from February to April 2020. Pregnant women in labor first consult at the health center where they receive prenatal care. When the pregnancy is at high risk or has indications for operation, the patients are transferred to the tertiary hospital, where assessment is made for spontaneous vaginal delivery versus caesarian section. After the operation, the patient is followed up postoperatively and discharged on day 3 or 4 postoperatively with an appointment for follow up in outpatient consultation several days later.

All pregnant women aged 15 years and above who delivered by cesarean section at $\mathrm{KRH}$ from January to March 2020 were enrolled, and all data were analyzed.

This study's primary objective was to determine the incidence rate of post-CS SSI, identify associated risk factors, as well as to characterize the most frequently involved microorganisms.

Data sources: Data were collected using a preestablished questionnaire to record patient demographics, clinical presentation and comorbidities, perioperative data. Follow up at 30 days post-operation was done using phone calls and registration records at post-operative scheduled hospital visits.

Quantitative variables: Variables were collected on patient's demographics, operative management, and the patient's outcome. We defined SSI as any infection occurring at the operated site within 30 days post-operation, as well as infection that involved the skin, subcutaneous soft tissue, any deep organ space. We considered "superficial infection" in all cases with infection in the subcutaneous tissue and above the fascia/; and "deep infection" as representing endometritis or intraperitoneal abscess.

The following were considered signs or symptoms of infection: pain or tenderness, localized swelling, redness, or heat.

Data management and statistical analysis: The questionnaire (data-collection tool) was explicitly designed for this study. The collected data was entered into a password protected Excel database and analyzed using SPSS version 21. Descriptive statistics were calculated and presented as frequencies and percentages. Univariate analysis was performed to determine the association between SSI and the other studied variables.

Ethical approval: No physical, social, emotional, legal and/or financial risks were identified.

Hospital ethical approval from the Kibungo Referral Hospital department of Maternity was obtained (No: KBG11/2020). 
Personal data was not used in the analysis. Each patient was assigned a unique study identifier number. A password-protected study identifier number linking with the personal identifier (name, hospital ID) were kept separately by the principal investigator (PI). Only the researcher and the research team had access to the study data and information.

An informed consent form was obtained from the patient or any other legally recognized attendant at the time of recruitment. Participation in the study was by choice and the patient had the right to withdraw from the study at any time during the study period. Funding \&Sponsors: No funding has been sought or gained for this project.

There were no incentives offered to patients whose data were used in this study.

\section{RESULTS}

We enrolled 201 patients who underwent caesarean section at Kibungo referral hospital aged 15 to 47 with a mean of 28 years. Eighty percent were married and $14 \%$ were single. The majority of the study participants had no underlying comorbidities (97\%), while only $3 \%$ had chronic conditions (hypertension, hepatitis, or diabetes mellitus). Fifty three percent (53\%) of performed operations were emergency and $47 \%$ elective. Repeat caesarean sections (41\%), obstructed labour or dystocia (24\%) and foetal distress (18\%) were the most common indications for operation. Povidone-iodine and chlorhexidine combined was the most common disinfectant used (81\%), whereas povidone-iodine alone was used to clean the operation site in $19 \%$. The duration of operation was between 30 to 45 minutes in $62 \%$ of the operated patients, followed by 46 to 60 minutes in $36 \%$, and just $2 \%$ lasted more than 60 minutes. Most of the operations were performed by general practitioners (92\%) versus $8 \%$ by obstetriciangynecologists (Appendix 2, page 44 and 45 ).

The incidence of post-CS SSI was 3.5\%, the most common being deep infection with 57\%, followed by superficial at $43 \%$. The mean days of hospital stay were 4 with a range of 2 to 15 days (Table 1 ). Obstructed labor/dystocia ((RR=4.55) at 95\% $\mathrm{Cl}$ [1.6-45.4]) increases the infection risk and showering prior to operation ( $(R R=0.39)$ at $95 \%$ $\mathrm{Cl}$ [0.005-0.29]) is associated with reduced risk of developing SSI while shaving 30 minutes prior to incision, the result shows that women who shaved are at 25.5 times risk of developing post-CS SSI (RR:25.5) at 95\% Cl [3.5-18.7] (Appendix 1, page 43).

Staphylococcus aureus represents the most common isolated microorganism of post-CS SSI at $57.1 \%$, followed by Escherichia coli with $28.6 \%$ and Klebsiella spp with $14.3 \%$.

\section{DISCUSSION}

Surgical infection remains the most challenging morbidity post caesarian section. This study found the Post-CS SSI incidence rate of 3.5\%, which falls within the worldwide range of 3\% to 15\% (Soule B.

Table 1: Incidence of post-CS SSI and other operative outcomes of the study participants

\begin{tabular}{|c|c|c|c|}
\hline Variable & & Observation (N) & Percentage (\%) \\
\hline \multicolumn{4}{|l|}{ Complications } \\
\hline & Surgical site Infection & 7 & 3.5 \\
\hline & Post-partum Hemorrhage & 4 & 2 \\
\hline & Anemia & 3 & 1.5 \\
\hline & Pneumonia & 3 & 1.5 \\
\hline & None & 184 & 91.5 \\
\hline \multicolumn{4}{|l|}{ Category of surgical site infection } \\
\hline & Superficial & 3 & 43 \\
\hline & Deep & 2 & 57 \\
\hline Unplanned Re-operation & Yes & 9 & 4 \\
\hline Length of hospital stay (Mean, range) in days & & & $4(2-15)$ \\
\hline
\end{tabular}


M., 2018). With reference to other published series in low- and middle-income settings, it remains low. Alfouzan et al. in 2019 found an incidence rate of $1.7 \%$ to $2.95 \%$ respectively between 2014 and 2016 in a study conducted in Kuwait, whereas Mpogoro and colleagues in 2014 found an incidence rate of $16.2 \%$ in their study conducted at Bugando medical centre, Tanzania $[10,12]$. This low incidence is likely due to good improvement in the practice of preventive measures of post-CS SSI. Among analyzed variables, showering prior to operation, shaving 30 minutes prior to incision, and Skin preparation were the identified key predictors of post-CS SSI, similar to other published studies $[13,14]$. These factors were significantly associated with the occurrence of post-CS SSI compared to other factors considered in this study (urgency of operation, indication, operation time, occupation, comorbidities, level of education of the woman and age).

The timing of shaving affects the wound infection rate. Shaving patients before surgery increases the risk of surgical site infections. The World Health Organization (WHO) has warned and urged health workers to avoid shaving surgical sites with a razor blade. When needed, especially if it interferes with the surgery, the global health body recommends using electrical clippers [15]. When shaving was done immediately before surgery, the infection rate was $3.1 \%$. Shaving 24 hours or more before surgery raised the infection rate to more than $20 \%$ [16].

The use of skin preparation solutions combining Povidone and Chlorhexidine was found to reduce the risk of occurrence of post-CS SSI. as compared to other published series, Ngai, et al.2015 in their study on skin preparation for prevention of SSI after C/S in hospital of US found that there is no significant difference in the use of either combined or single-skin preparation solution to reduce post-CS SSI; and Tetsuya Kawakita \& Helain J. Landy, 2018 in their study conducted at MedStar Washington Hospital Center found that there were few cases of post-CS SSI where chlorhexidine skin preparation was used $[17,18]$. There is variability in research findings, but the combination of povidone and chlorhexidine solutions and other prevention measures seem to reduce the risk of occurrence of post-CS SSI. Mihretu.M et al.2019 found that pregnancy-induced anemia, chorioamnionitis, midline skin incision, and post-operative hemoglobin less than $11 \mathrm{~g} / \mathrm{dl}$ are the only factors associated with post-CS SSI. In contrast, Cheng. K. et al., in their study on risk factors for surgical site infections in a teaching hospital, showed that comorbidity of diabetes and cancer contribute to the occurrence of post-CS SSI, which are different from our findings $[19,20]$.

Obstructed labor/dystocia was also significantly associated with infection [24]. This is similar to other published series [25]. Prolonged labor and multiple cervical examinations are well-known and significant variables associated with post-cesarean infection [26]

The most common microbial patterns in post-CS SSI are polymicrobial and due to gram-negative infections. In this study, most of the specimens isolated were monomicrobial and Staphylococcus aureus were the most isolates. One possible reason is that majority of our surgical site infections were superficial. This is supported by the study conducted by Shekhar P. et al. They found that, in 269 patients who develop SSI, $45.3 \%$ of them were related to Staphylococcus Aureus with a significant impact on their healing process [21]. This is different from the study conducted at the University teaching hospital of Kigali, Rwanda, which found that Klebsiella spp were the most isolated pathogens (55\%) [22]. The prior exposure to antibiotics before transfer to the hospital may also explain the monomicrobial patterns and impacted the ability to detect the microorganism. Prior studies have shown that $63.5 \%$ of patients receive antibiotics at the district hospital prior to transfer to a referral hospital [23]. However, in organ/space occupying $\mathrm{SSI}$, the pattern is polymicrobial gram-negative, as Kenneth et al.2018 found in their study on post CS peritonitis at the University Teaching Hospital of Kigali, Rwanda [11].

\section{CONCLUSION}

Usage of Skin preparation solution efficiently, not shaving $24 \mathrm{hrs}$ before CS, and showering one hour before CS were found to be highly associated with post-CS SSI when much caution is not taken, especially to women undergoing CS at the hospital. To maintain proper hygiene and adherence to available infection prevention measures reduces the incidence of SSI in post-CS patients. 


\section{REFERENCES}

[1] Khalid R Saeed BM, Incidence of surgical site infection following caesarean section: a systematic review and meta-analysis protocol.,BMJ OPEN 2017; . 5 (6): 1-8.

Bizimana JK, Prevalence and risk factors for post-cesarean delivery surgical site Int J Curr Microbiol App, 2016, 5(6):631-41.

[3] Soule B. M., Evidence-Based Principles and Practices for Preventing Surgical Site Infections., Joint commission international 2018, pp. 1-3.

[4] Del Monte N. A. MC, Postdischarge surveillance following cesarean section: the incidence of surgical site infection and associated factors, surgical site infection and associated factors 2010, 38(6):467-72.

[5] Jasim S. S. HH, Incidence and risk factors of surgical site infection among patients undergoing cesarean section, Clin Med Insights2017, 9:1-7.

[6] Jalil $\mathrm{MH} \mathrm{H}$. K., Surgical site infections following caesarean operations at a Jordanian teaching hospital: frequency and implicated factors, Sci Rep2017, (7)1:1-9.

[7] Vjosa A R. I. Zejnullahu, Surgical site infections after cesarean section, MBC infectious Diseases 2019, 7-8.

[8] Manisha Chhetry S. S., Risk factors for post caesarean surgical site infection at a tertiary care center in Eastern Nepal, Journal of College of Medical Sciences-Nepal 2017, (13) 3.

[9] Ahmed R K. G., Intra-abdominal infection followin cesarean section: a retrospective cohort study in tertial referral hospital, BMC 2019, 19:234. [10] Alfouzan M. A. W., Surgical site infection following cesarean section in a general hospital in Kuwait: trends and risk factors, Epidemiol Infect 2019, 147: e287.

[11] Kenneth R., Emerging threat to progress in maternal mortality reduction: Antimicrobial resistance trends among obstetric patient with post-cesarean peritonitis in Rwanda., East and central African journal of surgery 2018, 23(3):126200.

[12] Mpogoro FJ M. S., Incidence and predictors of surgical site infections following caesarean sections at Bugando medical Centre, Mwanza, Tanzania., Antimicrob Resist Infect Control 2014, 3(1):25.

[13] Túlio Cícero Franco Farret, Risk factors for surgical site infection following cesarean section in a Brazilian Women's Hospital: a case-control study, Brazilian Journal of Infectious Diseases 2015, 16784391.

[14] Rose AF F. B. M. J., Post-caesarean section surgical site infections: A retrospective audit and case note review at an Ethiopian referral hospital, oat open access test 2017, vol. DOI: 10.15761/ OGR.1000126.

[15] WHO, Global guidline fir the prevention for surgical site infection, Geneva, Switzerland: ISBN 978-92-4-155047-5, 2018.

[16] Sieczkowski C., Clipping not shaving intervention guidilines, Surgical site improvement program , 2014.

[17] Ngai I. M. M., Van Arsdale A. M. M., Govindappagari S. M., Judge N. E. M., Neto N. K. M., Bernstein J. M, Bernstein P. S. M. M. and Garry D. J. D., Skin Preparation for Prevention of Surgical Site Infection After Cesarean Delivery, Obstetrics and gynecology 2015, 126(6)1251-1257

[18] Landy H. J., Surgical site infections after cesarean delivery: epidemiology, prevention and treatment, BMC 2017, ISSN: 2054-958X, https:// doi.org/10.1186/s40748-017-0051-3.

[19] Mihretu Molla M. M, Surgical site infection and associated factors among women underwent cesarean delivery in Debretabor General Hospital, Northwest Ethiopia: hospital based cross sectional study, BMC 2019,ISSN: 1471-2393, https://doi. org/10.1186/s12884-019-2442-0.

[20] Cheng K, Li J, Kong Q, Wang C, Ye N, Xia $G$, Risk factors for surgical site infection in a teaching hospital: a prospective study of 1,138 patients, Dovepress 2015(9)1171-1177, https:// doi.org/10.2147/PPA.S86153.

[21] Shekhar Pal D. J., Staphylococcus aureus: A predominant cause of surgical site infections in a rural healthcare setup of Uttarakhand, Jounal of Family Medicine and primary care 20198(11): 3600-3606.

[22] Marie Josée Mukagendaneza, Incidence, root causes, and outcomes of surgical site infections in a tertiary care hospital in Rwanda: a prospective observational cohort study, MBC 2019, Vols. doi: 10.1186/s13037-019-0190-8.

[23] Ernest Muhirwa, Non-Obstetric Surgical Care at Three Rural District Hospitals in Rwanda: More Human Capacity and Surgical Equipment May Increase Operative Care, World J Surg 2016, 40(9):2109-16.

[24] Ayenew A. A, Incidence causes and maternofetal outcomes of obstructed labor in 
Ethiopia: systematic review and meta-analysis, BMC 2021, ISSN: 1742-4755.

[25] Shimelis H. S. F. A F, Incidence Causes and Outcome of Obstructed Labor in Jimma University Specialized Hospital, Ethiop J Health Sci 2010,
20(3): 145-151.

[26] Oliver C Ezechi A. E. H. A. C. V. G.-O. E. H, Incidence and risk factors for caesarean wound infection in Lagos Nigeria, BMC 2009, ISSN: 17560500 .

\section{Appendix 1: Association between the occurrence of post-CS SSI, demographic and clinical characteristics of participants}

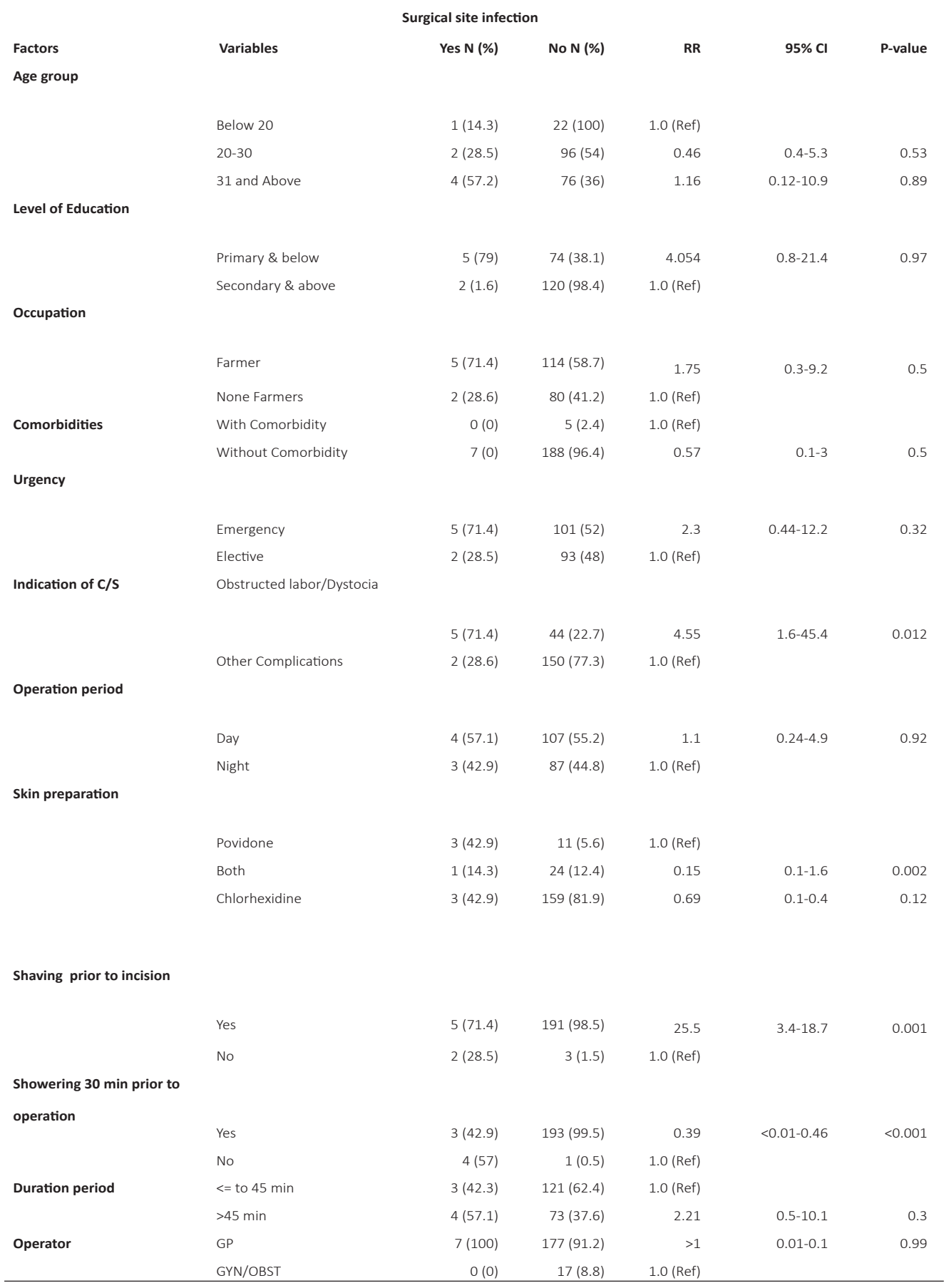

Ref: Reference group 
Appendix 2: Patients' demographic and clinical characteristics

\begin{tabular}{|c|c|c|c|}
\hline Variable & & N & Percentage (\%) \\
\hline \multicolumn{4}{|l|}{ Age (in years) } \\
\hline & Below 20 & 15 & 8 \\
\hline & $20-30$ & 107 & 53 \\
\hline & $31-40$ & 75 & 37 \\
\hline & 41 and above & 4 & 2 \\
\hline Mean age, range & & & $28(15,47)$ \\
\hline \multicolumn{4}{|l|}{ Residence } \\
\hline & In-zone & 180 & 90 \\
\hline & Out of zone & 21 & 10 \\
\hline \multicolumn{4}{|l|}{ Level of education } \\
\hline & Primary & 79 & 39.3 \\
\hline & Secondary & 94 & 47 \\
\hline & University & 28 & 14 \\
\hline \multicolumn{4}{|l|}{ Occupation } \\
\hline & Farmer & 119 & 59 \\
\hline & Gov/private employee & 65 & 32 \\
\hline & Student & 4 & 1 \\
\hline & None & 13 & 6 \\
\hline
\end{tabular}

Marital status

$\begin{array}{rrr}\text { Married } & 161 & 80 \\ \text { Single } & 29 & 14 \\ \text { Divorced } & 6 & 3 \\ \text { Widow } & 5 & 3\end{array}$

Comorbidities

$\begin{array}{rrr}\text { Hypertension } & 3 & 1.5 \\ \text { Hepatitis } & 2 & 1 \\ \text { Diabetes mellitus } & 1 & 0.5 \\ \text { None } & 195 & 97\end{array}$

Hospital stay prior to surgery 
Shaving before surgery

Type of prophylactic antibiotic

Urgency of CS

Indication for CS

Chlorhexidine and povidone-

iodine solutions

Chlorhexidine alone

Povidone alone

Operative period

Day

Night

Duration of operation

30 to 45 minutes

46 to 60 Minutes

Above 60 Minutes

Qualification of the operator

Obstructed labor/Dystocia

Fetal distress

Breech presentation

Multiple pregnancies

Macrosomia

Patient wish

Eclampsia
196

98

General practitioner

Obstetrician-gynecologist

Below 45 Minutes
200

99

1

53

47

95

41

24

18

5

3

3

3

2

1

162

81

25

12

14

7

11155

$90 \quad 45$

$124 \quad 62$

$73 \quad 36$

42

18492

$17 \quad 8$

124 